\title{
Research on the Reform of Experimental Teaching of Mechatronics Specialty Based on Social Demand
}

\author{
Guigen $\operatorname{Pan}^{1}$

\begin{abstract}
'Jiangxi Vocational Technical College Of Industry \& Trade, Jiangxi, Nanchang, 330038 739960103@qq.com
\end{abstract}

\begin{abstract}
In the process of applying mechatronics technology, the social demand puts forward more requirements for Mechatronics talents. In view of this phenomenon, this study focuses on the social needs of mechatronics, discusses and studies the problems existing in the experimental teaching process of Mechatronics specialty, and puts forward some measures to improve the experimental teaching system, establish and improve the evaluation system, optimize moral and intellectual education, and improve the experimental teaching of Mechatronics specialty, so as to better meet the social needs There will be demand.
\end{abstract}

Keywords: social needs, mechatronics, experimental teaching

\section{机电一体化专业实验教学以社会需求为基础的改革探 究 潘桂根 ${ }^{1}$}

\author{
${ }^{2}$ 江西工业贸易职业技术学院江西南昌 330103 \\ 739960103@qq.com
}

摘要:

在应用机电一体化技术的过程中，社会需求为机电一体化人才提出了更多要求。针对这种现象，本研究以机电 一体化的社会需求为中心, 展开讨论与研究, 通过对机电一体化专业实验教学过程中存在的问题进行阐述, 提 出了完善实验教学体系、建立健全评价体制、优化德育和智育教育等措施, 完善机电一体化专业实验教学, 从 而更好地满足社会需求。

关键词:社会需求; 机电一体化专业; 实验教学

\section{1 引言}

职业学校可以为我国技术型岗位提供更多技术 型的人才, 现阶段, 机电一体化技术得到了广泛的应 用, 许多职业学校选择设置这门学科。但是在进行机 电一体化教学的过程中, 因为学校并未全面掌握这类 技术的具体特点, 所以给教学工作带来了一定的负面 影响。另外, 现阶段社会对人才的需求持续增加, 机 电一体化专业的实践教学只有经历不断的优化、改革, 才能够更好地适应社会发展。所以, 基于社会需求的 机电一体化专业实验教学改革成为现今较热的话题, 应该怎样开展应用教学, 是职业学校机电一体化教师 函待思考与研究的问题。
2 机电一体化的社会需求与机电一体化专业人 才培养需求

\section{1 机电一体化的社会需求}

机电一体化的社会需求主要体现在以下几个方 面:

机电一体化, 即实现机械、电子、信息以及计算 机等多种技术的结合, 形成一门综合性的学术学科。 因此, 机电一体化的发展体现了它良好的技术性能, 并且在发展过程中已经被应用于很多领域, 所以形成 了广泛化、多元化的社会需求。 
因为机电一体化是一项具有强大综合性的技术, 要求从业人员熟练掌握多种技术的应用方法, 对于专 业的要求也非常严格, 因此人才非常欠缺, 社会需求 量也比较大。

随着社会的进步，中国的人才市场发展要求随之 不断变化, 在人才选取方面, 大部分企业偏向于实际 操作能力强、综合素质高的专业化人才。但是因为社 会市场人才要求逐渐提高, 让高职院校和学生需要从 自身的专业能力和综合素养为出发点进行发展, 从而 符合新时代人才选择的要求。

\section{2 机电一体化专业人才培养需求}

接下来我们探讨机电一体化专业人才的培养需 求。随着机电一体化技术和相关领域的不断进步, 大 部分现代科学技术逐渐出现在机电一体化行业生存 中, 这代表着机电一体化技术影响力不断扩大和其使 用价值不断提升。与此同时也促进了机电一体化技术 成为了社会发展的动力, 是不可或缺的组成部分。在 新时代的背景下, 在社会生产中机电一体化技术的使 用范围不断扩大, 并且为顺应时代发展潮流, 社会对 于机电一体专业化人才的要求也在不断提高。就“量” 来看, 如今在世界机械工业行业中机电一体化技术是 相关技术发展的方向, 也是中国机械工业进步的必经 之路。所以, 在机电一体化人才培养中缺少人才是当 今面临的难题。例如: 在 2015 年 6 月智联招聘发表 了北京市对于机电一体化专业人才需求的数据统计, 也就是在北京市对于机电一体化人才的需求量达到 每月 200 人, 并且这样只是智联招聘对于北京市所需 机电一体化专业人才的需求量。显而易见, 当代社会 对于机电一体化人才的需求量之大。就“质”来看, 机 电一体化技术的进步一定需要相关专业人才要求不 断提高, 提高专业化素养, 并且因为机电一体化专业 需要较强的实际操作能力, 所以机电一体化相关领域 对于技术应用型人才、复合型人才的需求较多, 同样 这样代表了学校应该重视机电一体化专业的理论知 识教学和实践教学, 从而强化学生的综合素养。从而 推动机电一体化专业教学适应社会的进步。

\section{3 机电一体化专业实验教学中出现的问题表现}

\section{1 重视理论授课, 轻视实践教学}

机电一体化传统的教学方式主要是指教师在讲 台上利用一块黑板和教师手中的教材对学生授课。但 是就机电一体化专业教学来说, 机电一体化技术是十 分注重实践教学的, 教材中的知识多为原理和概念, 教学内容复杂多变, 从而导致了学生在教师的教学中 难以真正的了解到机电一体化专业中的技术重点, 使 学生在实际操作容易出现偏差。再者企业对于机电一 体化技术的操作能力比学校的要求更高, 从而会增加 学生往后的日常工作的难度, 并且职业院校也难以满 足社会对于专业化人才的需求量。

\section{2 实验教学的体系尚不完善}

在机电一体化的教育教学实践中, 实验教学的水 平还处在一个不成熟的时期。在机电一体化专业的学 生开展实验的时候, 教师普遍是实验课堂中的主体。 学生的工作单单是复制教师所做出的实验环节, 只是 让学生具有机械操作的经验, 学生并没有深度地思考 实验步骤的原因, 也没有对实验步骤中需要注意的细 节进行关注。在实践中, 学生缺少创新性思维的发散, 在以后的工作中也会缺少创新探索精神。就现在机电 一体化不断发现的状况来看, 学生继续在死板沉问的 课堂氛围中学习, 日后在人才市场上就缺少相应的竞 争实力。另外, 实验教学体系不完善从另一方面体现 在职业学校有限的实验器材方面, 对相关的实验环节, 学生只能通过课堂观看的方式了解, 不能实际的操作, 即便是有相关器材供学生实践操作, 其时间也是极其 有限的。

\section{3 学生素质较低, 不符合就业标准}

步入二十一世纪以后, 我国社会不断发展, 社会 对机电一体化人才提出了更高的要求。但现阶段大部 分高职院校在培育机电一体化人才时, 只注重提高学 生某一方面的能力, 比如: 计算机技术、电力电子技 术, 而不注重提高学生的综合能力。除此之外, 职业 学校在培养学生综合素质的过程中, 只注重提高专业 的就业率, 为学生设立了大量的专业课程, 没有意识 到素质教育的重要性。这些不足会导致学生的素质较 低, 不符合当今社会的就业标准, 对学生的就业产生 不利影响。

\section{4 机电课堂的评价机制不够完善}

科学、完善的课堂评价机制在教学过程中起着十 分重要的作用, 它可以使教师了解学生的学习情况和 学习态度, 使学生正确认识到自己的学习水平。虽然 传统的评价方式可以反映出学生对课堂知识的掌握 情况, 但无法反映出学生在课堂上的学习状态和学生 的真实水平, 无法充分发挥出评价机制的价值。因此, 教师要不停的依靠评价机制开展考核工作, 依靠更加 具有系统程度的评价机会, 让教师最大程度的了解学 生能力的变动情况, 并具体分析教育教学方法存在的 缺点, 让学生认识到自己的学习水平, 利用好自身的 优点。

\section{4 基于社会需求的机电一体化专业实验教学改 革的措施}

\section{1 完善实验教学的体系}

在机电一体化实验教学中需要进行以下几点的 安排。第一, 开展全面的教学, 在教育教学实践中, 考虑不同专业所学的知, 对其开展有针对程度的实践 教学。比如, 在机械学科中开展实践教学, 要将重点 
放在物理实验、力学实验、流体力实验以及机械设计 实验等相关实验上; 在电力实验教学方面, 可以严格 区分电工实验和电子实验就可以; 在控制类实验教学 方面, 要重点开展单片机实验、PLC 实验和电机实验; 在综合实验教学方面, 可以开展机器人实验和数控实 验。依靠各种各样的实际实验教学工作, 让学生在具 体的实践中达到理论和实验的一体化。第二, 增加职 业学校在机电一体化实验室上的资金投入数量, 把实 验室具体地分成三个部分：基础实验室、综合实验室 和数控机床等高级实验室, 学生还可以采用校企结合 的教育教学模式, 寻找相关企业给学校更多的设备和 资金支持, 依靠这样的教育教学模式, 不但可以提高 实验室的经济实力, 还能给学生提供一定的就业机会。 第三, 提高教师的实验教学水平, 在具体的教育教学 实践中, 教师要把学生作为教学的主体, 让学生在实 践中达到理论知识和实验的具体结合, 最大程度的发 挥出自己的发散性思维, 提升创新能力。

\section{2 增强德育和智育教育水平}

提升学生素养必然离不开智育和德育两个方面。 “你的专业知识决定你是否能够从事这个行业, 你的 做人水平决定你能够在这个行业走多久。”所以, 增强 德育和智育水平对促进学生全面发展有重要意义。在 智育方面, 可以采用理论与实践结合、综合性专业教 学等方式, 增强学生的专业知识掌握程度。教师还要 对考评机制进行改革, 可以增加学生的参与性。在德 育方面, 职业院校要合理安排德育教育的时间, 为学 生提供志愿服务等社会实践活动。让学生在理解德育 思想的同时, 切身体会德育的实践意义。德育和智育 教育要双管齐下, 不仅能够提升学生的综合素养, 还 能提升学生的竞争力, 在未来就业中, 学生的社会竞 争力会强于其他毕业生。

\section{3 加快评价体制的建立健全进程}

考核评价机制的制定主要是针对机电一体化的 教学展开的。机电一体化教学的主要特性为理论与实 验结合，针对此特点可以将考核评价机制分为两部分。 一部分的评价根据主要为课程的理论学习情况。学生 的上课态度、课堂参与度、笔试考试成绩等都将作为 教师评价的依据。另一部分的评价根据学生的实验学 习情况。教师对学生在实验课堂的表现态度、深入思 考能力、分析问题能力进行综合评价。最后再将两者 综合评价结合起来, 得出最终评分。另外, 教师还可 以设立奖惩制度来作为考核评价机制的一部分, 在考 核完成后, 对最终评分优秀、某一领域表现突出的学 生进行奖励, 对最终评分不达标的学生进行一定的惩 罚。增强学生的学习主动性和积极性。

\section{4 产学结合的人才培养模式}

为适应社会需求, 机电一体化教学改革的有效途 径之一为推动学生进入企业参与生产实践。所以, 在
机电一体化专业人才培养中, 教学模式要实现多元化 发展, 将课堂教学和产学紧密结合起来, 让学生感受 实际的工作氛围和环境, 体验真实的工作过程。对企 业来说, 产学结合的人才培养模式, 既能为企业提供 更好的人才选择，又能让企业体验高校的科研、师资 资源。对学校来说, 产学结合的人才培养模式不仅能 为机电一体化专业学生的创造实习实践的机会, 而且 也能缓解学校实践机会匮乏的局面。所以, 产学结合 主体可以让企业和学校更好的合作, 并促进机电一体 化专业教学与时俱进。

\section{5 打造双师型的师资队伍}

为提高机电一体化专业的教学质量, 职业学校注 重加强师资队伍的综合能力, 由于机电一体化专业具 有较强的实践性和实用性, 所以职业学校要提高教师 的专业素养和实践能力。步入二十一世纪以后, 随着 社会的不断发展, 社会对机电一体化人才提出了更高 的要求。为了满足社会需求, 职业学校要加强师资队 伍的力量, 注重提高教师的专业能力, 只有这样才可 以保障机电一体化专业的教学质量, 培养出更多高素 质的综合型机电一体化人才。教师机电一体化教学过 程中起着十分重要的作用, 机电一体化专业教师要担 负起自身的责任, 将技能教学融入到理论教学中, 注 重提高学生的专业能力和实践能力, 满足学生在学习 过程中的不同需求。除此之外, 学校也要为教师提供 更多的学习机会, 通过多种方式来培训机电一体化专 业教师, 以此来提高机电一体化专业教师的专业能力 和实践能力, 提高机电一体化专业的教学质量。

\section{5 结论:}

步入二十一世纪以后, 我国社会不断发展, 社会 对机电一体化人才提出了更高的要求。为满足社会需 求, 职业学校需要改革机电一体化专业实验教学模式, 建立起完善的实验教学机制, 注重对学生的素质教育 和智育教育。同时, 学校还要不断完善课堂评价体系, 加强师资队伍的综合能力, 以此来提高机电一体化专 业的教学质量, 提高学生的专业能力和实践能力, 使 学生在就业时具有较强的竞争力。

\section{REFERENCES}

[1] Tang Zhongyan, Han Qingyao, Zheng Haiming, Bai Jie, Li Xin. Application of system experiment method in mechatronics practice teaching $[\mathrm{J}]$. China Electric Power Education: I, 2011 (12): $139-140$

[2] Wang Zhen, sun Qun, Zhao Ying. Research on teaching reform of mechatronics technology course for mechanical specialty in local colleges and universities $[\mathrm{J}]$. Science and education guide (Electronic Edition), 2015,0 (33): 73-74 
[3] Li Guohua, Qian Yiqiu. Analysis on the leading role of vocational college skills competition in the teaching reform of numerical control technology specialty $[\mathrm{J}]$. Higher Vocational Education: Journal of Tianjin Vocational University, 2012 (3): 65-67

[4] Yu Nianjun. Analysis on the practical ability of cultivating the contestants of Mechatronics skills competition -- Taking Danyang secondary vocational school in Jiangsu Province as an example [J]. Southern agricultural machinery, 2019,50 (23): 229-229

[5] Yang Xiaoyong. Modular practice teaching reform of Mechatronics Technology Specialty in Higher Vocational Colleges under the background of intelligent manufacturing $[\mathrm{J}]$. Science and Education Guide: electronic edition, 2020 (24): $157-158$

[6] Cheng Gang, Jiao Shangbin, Li Shengmin, sun Xuxia, Liu Han, Zheng Chaoyang. Design and debugging of speed regulation system experimental platform based on s7-200smartplc [J]. Experimental technology and management, 2019,36 (3): 116-120

[7] He Meigui. Practical teaching curriculum reform of Mechatronics specialty in Higher Vocational Colleges Based on the combination of work and study [J]. Journal of Anhui Metallurgical Technology Vocational College, 2017, 27 (2)

[8] Yu Delin. Teaching reform and practice of mechatronics technology under the guidance of skills competition [J]. Management and technology of small and medium enterprises, 2015, (27)

[9] Meng Xianhui, an Jiufu, Zhao Chenguang, Liu Zhigang. Exploration and practice of improving teaching quality of measurement and control instrument design course $[\mathrm{J}]$. Industry and Technology Forum, 2014,0 (17): 146-147

[10] Xiong Xiaoqin, Xie Dan, ye Dameng, Liu Yifan. Reflections on the teaching reform of "mechatronics technology" course [J]. China Electric Power Education: middle, 2013 (3): 3334

[11] Fang Guisheng, Sun Ping. Making full use of Opto Mechatronics training device to cultivate students' comprehensive ability $[\mathrm{J}]$. Laboratory research and exploration, 2011,30 (1): 194-198

[12] Wang Tianbao, Cheng Weidong. Research and practice of innovative engineering talents training mode based on CDIO -- Practice of engineering education reform in Chengdu University of information engineering $[\mathrm{J}]$. Research on higher engineering education, 2010 (1): 25-31

[13] Shao Yuanyuan, Guan Tao. Exploration and practice of Electromechanical Integrated Experimental Teaching Reform [] China modern educational equipment, 2010 (9): 84-86

[14] Zhao Yan, Ruan Xiang FA. Exploration and practice of the construction of electromechanical integrated laboratory $[\mathrm{J}]$. Laboratory research and exploration, 1999,18 (4): 107-109

[15] Lu Qian, Wang Fuyuan, Cui Zhi, et al. Discussion on the reform strategy of practice teaching of mechatronics and physicochemical courses in Colleges and universities [] science and education guide, 2011 (25): 156-157.

[16] Song Weihai, Zhang Huirong. Discussion on the fine construction of professional courses based on professional needs -- Taking Mechatronics major in Higher Vocational Colleges as an example [J]. Journal of Shandong Agricultural Engineering College, 2016,33 (5): 27-31. 